\section{Homogamy for Birthplaces and Cultural Diversity}

\author{
Kohei Tamura \\ Department of Biological Sciences, the University of Tokyo, Hongo 7-3-1, \\ Bunkyoku, Tokyo 113-0033, Japan \\ Author for correspondence (tamura $(a$ biol.s.u-tokyo.ac.jp)
}

A major goal of anthropology is to understand the forces maintaining cultural variation. Although theoretical studies have proposed various mechanisms that may homogenize cultural diversity, recent empirical studies have suggested that there is considerable within-population cultural variation. In the present study, by analyzing marriage patterns in Japan in 1967 , I show that the degree of homogamy in a prefecture was significantly affected by the diversity of birth prefectures of the inhabitants, indicating that a more culturally diverse prefecture might be more likely to resist the evolutionary forces that homogenize cultural diversity.

\section{Keywords}

mate choice, geographical variation, marriage

\section{Introduction}

Human culture exhibits enormous variation both within and between societies. Recent empirical studies have demonstrated that cultural variation between populations can be much greater than genetic differences (e.g., Bell, Richerson, \& McErleath, 2009). Theoretical studies have proposed various factors that homogenize within-population cultural diversity, leading to cultural differentiation between populations. For example, models of cultural blending, where each pupil acquires the average of cultural traits exhibited by its exemplars, showed that cultural blending reduces the amount of cultural variation in a population (Boyd \& Richerson, 1985; CavalliSforza \& Feldman, 1981). Based on these results, some researchers have argued that there is a greater potential for group selection on culture than genes (cultural group selection). This argument posits that human cultures exhibit low levels of within-population diversity owing to processes such as conformity that can lead to the fixation of particular cultural variants, resulting in higher levels of between-group diversity (Boyd \& Richerson, 1985).

However, it has also been reported that there is a considerable amount of cultural variation within populations: although cultural variation between populations can be much higher than genetic differences, much of the overall variation is within populations (Bell et al., 2009; Ross, Greenhill, \& Atkinson, 2013). Lamba and Mace (2013) cast doubt on cultural conformity by showing within-group variation in behaviors in economic games. It has not been fully understood how cultural variation within and between populations has been maintained under the evolutionary forces that homogenize cultural diversity.

The homogenizing effects of migration or cultural blending on cultural variation may have been overestimated. By analyzing friendship networks in schools in the United States, Moody (2001) demonstrated that racial segregation in friendships peaks at a moderate level of racial heterogeneity. McErleath, Boyd, and Richerson (2003) developed a mathematical model, suggesting that ethnic differences can be strongest at boundary regions between different cultural groups. These studies have indicated that increase in interaction between individuals with different cultural backgrounds can result in the emergence of cultural boundaries rather than lead to cultural blending.

Marriage patterns have been suggested as a key factor in shaping both genetic and cultural structures (e.g., Cavalli-Sforza, 2000). Evidence for homogamy, or marriage between individuals who are similar to each other, has been found for a wide variety of physical and cultural traits (Cavalli-Sforza \& Feldman, 1981; Nojo, Ihara, Furusawa, Akamatsu, \& Ishida, 2011; Nojo, Tamura, \& Ihara, 2012; Seki, Ihara, \& Aoki, 2012). Homogamy may play a role in the maintenance of cultural variation within populations. Sociologists have reported slow cultural assimilation of immigrants, which is at least partly caused by homogamy for ethnic and religious cultural traits. Bisin and Verdier (2000) investigated the effect of homogamy on cultural diversity by developing a mathematical model in which homogamous families are more successful in vertical transmission than culturally mixed families are. As noted above, interaction between individuals with different cultural backgrounds can result in the emergence of cultural boundaries and, consequently, promote population subdivision (McErleath et al., 2003; Moody, 2001). It may be reasonable that marriage patterns are also affected by ethnic or cultural heterogeneity: population mixing can enhance segregation in marriage, resulting in a higher level of homogamy. Therefore, a more culturally diverse population may be more likely to resist the homogenizing effects of cultural blending on cultural diversity, especially of cultural traits that are vertically transmitted.

Usui, Katabami, and Kaneko (1970) collected data on marriages in 1967 in Japan and pointed out that married couples tend to be from the same prefectures, that is, there is homogamy for birth prefecture. Their data set provides an opportunity for investigating factors affecting the degree of homogamy. As previous studies showed (Bisin \& Verdier, 2000; Moody, 2001), ethnic or cultural diversity is a possible candidate. In the present study, using the 
diversity of birth prefectures of inhabitants in a given area as an indirect measure of that area's ethnic or cultura diversity, I examine whether the degree of homogamy is sensitive to the diversity of birth prefectures.

\section{Material and methods}

Relevant marriage data were obtained from Usui, Katabami, \& Kaneko (1973a, 1973b, 1973c, 1973d) for 46 prefectures in Japan (excluding Okinawa prefecture because of data availability). They collected marriage registration cards presented mainly from August to December in 1967. Each card was classified according to honseki, or the place registered in the Japanese family registry, of the bride and bridegroom. Hereafter, honseki is assumed to be identical to the birth prefecture. Since it is difficult to obtain regionally disaggregated data on marriage, I treated each prefecture as a regional population for the sake of analysis. The data set includes the number of marriages registered in prefecture $k$ between brides from prefecture $i$ and bridegrooms from prefecture $j$.

The diversity of birth prefectures in prefecture $k$ is given by $1-\sum_{i=1}^{46} p_{i, k}^{2}$, where $p_{i, k}$ is the frequency of individuals from prefecture $i$, whose marriage is registered in prefecture $k$. The index, called heterozygosity, is a commonly used measure of genetic diversity in a
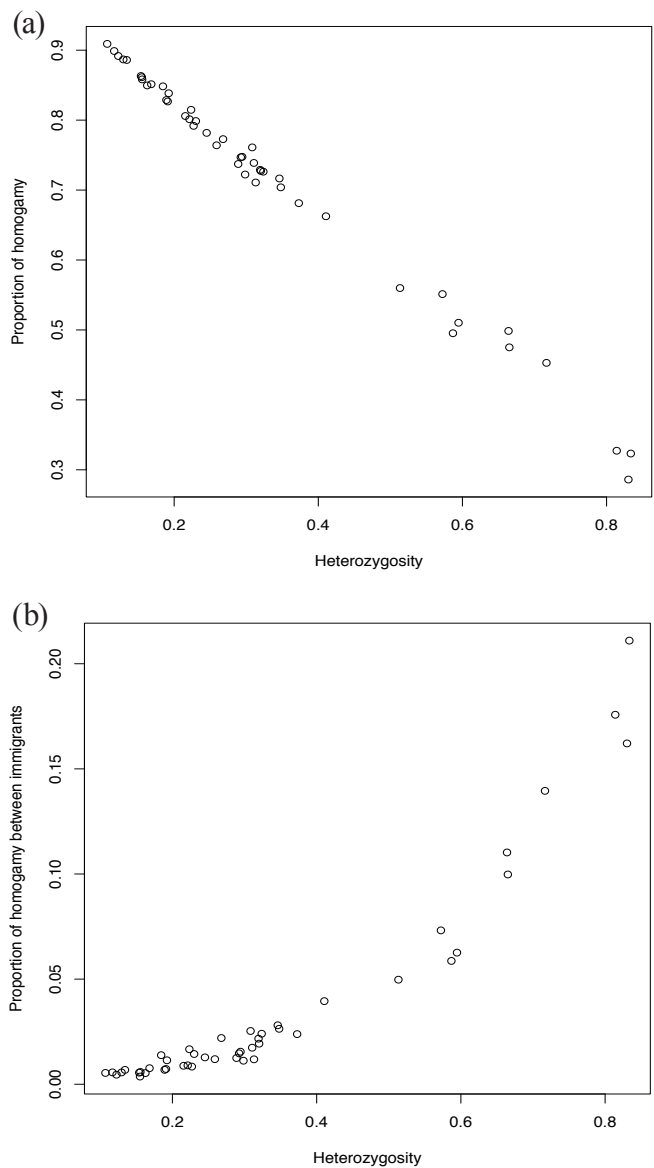

Figure 1. (a) The proportion of homogamy among all inhabitants, plotted against the degree of heterozygosity. (b) The proportion of homogamy between immigrants from the same prefectures among all marriages of immigrants in a prefecture, plotted against the degree of heterozygosity. Each circle represents a prefecture $(n=46)$. population (Hartl \& Clark, 2006). Heterozygosity measures the probability that two randomly selected individuals are from different prefectures.

In a prefecture where immigrants are rare, the proportion of homogamy for birth prefectures can be very high without there being any preference for samecharacteristic partners. Figure 1(a) demonstrates that the proportion of homogamy among all marriages in a prefecture becomes lower as the degree of heterozygosity increases, although the opposite is true for the proportion of homogamy between immigrants among all marriages of immigrants in a prefecture (Figure 1(b)).

To control for the relative opportunity for marriages between individuals from different prefectures, for each prefecture, the ratio of observed to expected numbers of homogamous marriages (hereafter called $\mathrm{O} / \mathrm{E}$ ratio of homogamy) is calculated, which measures how the observed marriages deviate from random mating expectation owing to various factors including mating preference and spatial and/or social structures.

To examine whether the degree of heterozygosity affects the $\mathrm{O} / \mathrm{E}$ ratios of homogamy, linear regression analysis was conducted. All statistical analyses were performed using R Version 2.15.3 (R Development Core Team, 2013).

\section{Results}

Figure 2 shows the O/E ratios of homogamy, plotted against the degree of heterozygosity, suggesting that the degree of homogamy becomes higher as the degree of heterozygosity increases.

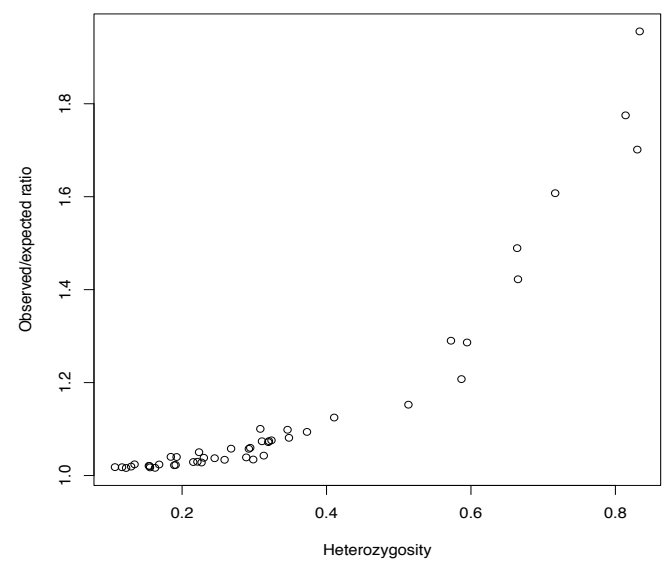

Figure 2. The ratios of observed to expected numbers of homogamous marriages, plotted against the degree of heterozygosity. Each circle represents a prefecture $(n=46)$.

All of the $\mathrm{O} / \mathrm{E}$ ratios of homogamy are greater than one, indicating that married couples are more likely to be from the same prefectures than expected from random

Table 1. The results of linear regression analysis

\begin{tabular}{lccc}
\hline Independent variable & Estimate & S. E. & $P$-value \\
\hline Heterozygosity & 0.9982 & 0.0640 & $<0.001$ \\
\hline
\end{tabular}


mating.

Table 1 summarizes the results of the linear regression analysis, suggesting that the degree of heterozygosity significantly affects the degree of homogamy.

\section{Discussion}

In the present study, by analyzing marriage patterns in 1967 in Japan, I demonstrated that the degree of homogamy was significantly affected by the degree of heterozygosity, or the diversity of birth prefectures of inhabitants. I also showed that the proportion of homogamy between immigrants becomes higher as the degree of heterozygosity increases. My results indicate that by inhibiting cultural and marital assimilation of immigrants, a more culturally diverse prefecture may be more likely to resist the evolutionary forces that homogenize cultural diversity.

Although these results identified the marriage patterns that were expected according to previous studies (Bisin \& Verdier, 2000; McErleath et al., 2003; Moody, 2001), because of the difficulty in delineating cause-and-effect relationships, the present study cannot control for the effects of marriage patterns on the diversity of birth prefectures of inhabitants and other social environments. Developing sophisticated mathematical and statistical models of homogamy may be a possible direction for future research to reveal interrelationships between marriage patterns and social environments.

While my results suggest that the degree of homogamy monotonically increases as the degree of heterozygosity increases, this might be due to limited sample availability. Moody (2001) demonstrated that racial segregation in friendships peaks at a moderate level of racial heterogeneity, suggesting that if the degree of heterozygosity exceeds the range I considered, the degree of homogamy might decrease.

In the present study, I used marriage data obtained from Usui et al. (1973a, 1973b, 1973c, 1973d). Since they collected almost all marriage registration cards presented from August to December in 1967 in Japan, it is unlikely that members of specific social groups were more or less likely to be included in the sample. However, as the number of marriages in Japan has varied by season (Statistics and Information Department, Minister's Secretariat, Ministry of Health, Labour and Welfare, 2005), if there were drastic seasonal changes in marriage patterns, some of the results of the present study may require modification.

McErleath et al. (2003) argued that interaction between different cultural groups could promote ethnic differences. Although my results are in line with the prediction by McErleath et al.'s (2003) model, because data was unavailable on the places where couples first met, I was unable to identify the proximate mechanisms, including preference for cultural similarity, spatial structure, and economic factors such as group employment. Experimental studies may complement the limitations of the present study and prove fruitful in increasing our understanding of the nature of human mating preferences.

Since much cultural competition is likely to occur on a village scale, a prefecture is composed of a set of subpopulations that are the unit upon which cultural group selection can work. Because I used regionally aggregated data, the relative magnitude of cultural variation within and between the subpopulations is unclear, indicating that the results of the present study may have limitations in their application to cultural group selection. Despite the limitations, if the results hold for smaller-scale societies, the present study may have some implications for cultural group selection. Although a theoretical argument against group selection is based on difficulties in the maintenance of variation between subpopulations under homogenizing forces of migration or other forms of population mixing, Boyd and Richerson (1985) argued that conformity transmission can mitigate the force of population mixing, resulting in a greater potential for group selection on culture than gene. My results may indicate that because homogamy can inhibit cultural assimilation of immigrants, conformity transmission may be less likely to maintain between-group cultural differences in the presence of the high degree of homogamy in culturally diverse subpopulations. However, on the contrary, the high degree of homogamy may promote cultural group selection, because a subpopulation that harbors two or more different cultural groups eventually leads to population subdivision, resulting in smaller culturally homogeneous subpopulations. In sum, various factors can affect whether homogamy promotes or inhibits cultural group selection.

While mathematical models of cultural group selection, as well as those of genetic group selection, have supported the idea that inter-group conflict can promote the evolution of cooperation, the potential contribution has been discussed (Bell et al., 2009; Boyd, Gintis, Bowles, \& Richerson, 2003; Lamba \& Mace, 2013; Tamura, Morita, $\&$ Ihara, 2011). Further theoretical and empirical research is clearly needed to investigate interrelationships among various mechanisms that are necessary for cultural group selection to be effective and, as a result, what geographical patterns of cultural variation can emerge.

\section{Acknowledgement}

I would like to thank Y. Ihara and an anonymous reviewer for their helpful comments. This work is supported by Grant-in Aid for JSPS fellows.

\section{References}

Bell, A. V., Richerson, P. J., \& McElreath, R. (2009). Culture rather than genes provides greater scope for the evolution of large-scale human prosociality. Proceedings of the National Academy of Sciences of the United States of America, 106, 17671-17674. (doi: 10.1073/pnas.0903232106)

Bisin, A., \& Verdier, T. (2000). "Beyond the melting pot": Cultural transmission, marriage, and the evolution of ethnic and religious traits. Quarterly Journal of Economics, 115, 955-988. Cdoi: $10.1162 / 003355300554953)$

Boyd, R., \& Richerson, P. J. (1985). Culture and the evolutionary process. Chicago: University of Chicago Press.

Boyd, R., Gintis, H., Bowles, S., \& Richerson, P. J. (2003). The evolution of altruistic punishment. Proceedings of the National Academy of Sciences of the United States of America, 100, 3531-3535. (doi: 10.1073 pnas.0630443100) 
Cavalli-Sforza, L. L. (2000). Genes, peoples, and languages. New York: North Point Press.

Cavalli-Sforza, L. L., \& Feldman, M. W. (1981). Cultural transmission and evolution: A quantitative approach. Princeton: Princeton University Press.

Hartl, D. L., \& Clark, A. G. (2006). Principles of population genetics (4th ed.). Sunderland: Sinauer Associates.

Lamba, S., \& Mace, R. (2013). The evolution of fairness: Explaining variation in bargaining behaviour. Proceedings of the Royal Society B: Biological Sciences, 280, 20122028. doi: 10.1098 rspb.2012.2028)

McErleath, R., Boyd, R., \& Richerson, P. J. (2003). Shared norms and the evolution of ethnic markers. Current Anthropology, 44, 122-129. (doi: 10.1086/345689)

Moody, J. (2001). Race, school integration, and friendship segregation in America. American Journal of Sociology, 107, 679-716 (doi: 10.1086/338954)

Statistics and Information Department, Minister's Secretariat, Ministry of Health, Labour and Welfare. (2005). Vital statistics of Japan [CD ROM]. Health, Labour and Welfare Statistics Association.

Nojo, S., Ihara, Y., Furusawa, H., Akamatsu, S., \& Ishida, T. (2011). Facial resemblance and attractiveness: An experimental study in rural Indonesia. Letters on Evolutionary Behavioral Sciences, 2, 9-12. doi: 10.5178/lebs.2011.11

Nojo, S., Tamura, S., \& Ihara, Y. (2012). Human homogamy in facial characteristics: Does a sexualimprinting-like mechanism play a role? Human Nature, 23, 323-340. (doi: 10.1007/s12110-012-9146-8)

R Development Core Team. (2013). R: A language and environment for statistical computing, Version 2.15.3. Vienna: R Foundation for Statistical Computing.

Ross, R. M., Greenhill, S. J., \& Atkinson, Q. D. (2013). Population structure and cultural geography of a folktale in Europe. Proceedings of the Royal Society B: Biological Sciences, 280, 20123065. (doi: 10.1098 rspb.2012.3065)

Seki, M., Ihara, Y., \& Aoki, K. (2012). Homogamy and imprinting-like effect on mate choice preference for body height in the current Japanese population. Annals of Human Biology, 39, 28-35. doi. 10.3109/03014460.2011.635695)

Tamura, K., Morita, R. C., \& Ihara. Y. (2011). Evolution of egalitarian punishment. Letters on Behavioral Evolutionary Sciences, 2, 20-23. (doi: 10.5178 lebs.2011.14)

Usui, T., Katabami, J, \& Kaneko, I. (1970). Statistical studies on the origin of the birth-place of the couple (Tsukonken). Bulletin of the Institute of Public Health, 19, 197-206. (in Japanese)

Usui, T., Katabami, J, \& Kaneko, I. (1973a). Tabulations of first marriage registrations with place of honseki, part 1. Bulletin of the Institute of Public Health, 22, 29-53. (in Japanese)

Usui, T., Katabami, J, \& Kaneko, I. (1973b). Tabulations of first marriage registrations with place of honseki, part 2. Bulletin of the Institute of Public Health, 22, 83107. (in Japanese)

Usui, T., Katabami, J, \& Kaneko, I. (1973c). Tabulations of first marriage registrations with place of honseki, part 3. Bulletin of the Institute of Public Health, 22, $177-$ 201. (in Japanese)

Usui, T., Katabami, J, \& Kaneko, I. (1973d). Tabulations of first marriage registrations with place of honseki, part 4. Bulletin of the Institute of Public Health, 22, 245265. (in Japanese) 